\title{
Laughlin states and their quasi-particle excitations on the torus
}

\author{
Martin Greiter, Vera Schnells, and Ronny Thomale \\ Institute for Theoretical Physics, University of Würzburg, Am Hubland, 97074 Würzburg, Germany
}

\begin{abstract}
We provide a full derivation of Laughlin's Jastrow-type wave functions for quantized Hall states subject to periodic boundary conditions using an operator formalism. The construction includes the quasi-hole and the technically more challenging quasi-electron excitation, which was left as an open problem in the classic paper by Haldane and Rezayi [Phys. Rev. B 31, 2529 (1985)].
\end{abstract}

PACS numbers: 73.43.Cd, 03.65.Vf, 02.30.Fn

\section{INTRODUCTION}

Laughlin's theory of the fractional quantized Hall effect $^{1}$ has been arguably one of the most influential development in theoretical condensed matter physics in the past decades. It has been generalized to other rational filling odd-denominator Landau level fractions through a hierarchy of fractionally quantized Hall states ${ }^{2-5}$, but also to even denominator filling fractions through inclusion of $\mathrm{p}$-wave pairing correlations ${ }^{6-8}$. The fractionally charged quasi-particle excitations of the odddenominator states are the first realizations of particles obeying fractional statistics ${ }^{9,10}$ in two space dimensions (Abelian 2D anyons), while the quasi-particles in the paired odd-denominator states and their generalizations obey non-Abelian statistics ${ }^{6,11-13}$ (non-Abelian 2D anyons). The concept of non-Abelian statistics, which now plays a preeminent role in the field of quantum computation $^{14,15}$, was actually discovered in paired Hall states $^{6}$, where it is understood in terms of unpaired Majorana fermions ${ }^{11,16}$ at the quasi-particle vortex cores. Wave functions similar to the Laughlin and Read-Rezayi series ${ }^{17}$ of quantized Hall states describe the exact ground states of an integrable spin one-half chain model ${ }^{18,19}$ and its generalizations to critical models for higher spin chains $^{20,21}$. These models have led us to understand Abelian $^{22,23}$ and non-Abelian anyons in one dimension ${ }^{20}$. The concept of topological order ${ }^{24-26}$ was introduced in the context of the Abelian chiral spin liquid ${ }^{27-30}$, which is a spin liquid in which bosonic spin flip operators are described by a Laughlin-Jastrow-type Hall wave function.

Laughlin's wave functions ${ }^{1,31}$, as originally proposed, describe circular droplets of fractionally quantized Hall fluids in the open plane, the geometry Laughlin had used for early numerical work. For the definite confirmation of its correctness, Haldane eliminated the boundary by reformulating the theory on a sphere in a magnetic monopole field ${ }^{2,32,33}$, and then showed numerically that the energy gap in the excitation spectrum remained intact as the Hamiltonian is continuously varied from screened Coulomb interactions to the parent Hamiltonian for the Laughlin states. The third important geometry is the torus or periodic boundary conditions, the only among them with a non-zero genus $(g=1)$, and hence the only one in which the topological degeneracies originally discovered by Haldane and Rezayi ${ }^{34}$ are observable.
These degeneracies have then been interpreted as a manifestation of topological order, a new concept introduced by Wen ${ }^{24,25}$ to describe order in quantum Hall and spin liquid states. All of this has been understood in the early years, and has now been well established for decades.

There is one small, technical aspect in the formulation of Laughlin's theory, however, which was not resolved by the masters. Wave functions for the Laughlin states and the quasi-hole excitations were given by Haldane and Rezayi $^{34}$, but formulating the technically more challenging quasi-electron excitation was left as an open problem. The formulation of ground states and quasi-holes was subsequently generalized to include Pfaffian states on the torus ${ }^{35}$, but then again, the quasi-electron excitations were not addressed. Furthermore, an elegant formulation of quantized Hall wave functions in terms of conformal correlators was developed ${ }^{6,36,37}$. While quantum Hall ground states were generalized to the torus for composite fermions and the Haldane-Halperin hierarchy through conformal field theor $\mathrm{y}^{38-40}$, an explicit formulation of Laughlin's quasi-electron wave function has so far only been reported in the open plane ${ }^{41}$.

Presumably, there are two reasons for this. First, it is highly non-trivial from a technical point of view. Haldane and Rezayi ${ }^{34}$ devoted a paragraph to the problem in their classic paper, but the form of an Ansatz they suggest is already inconsistent with the results we present below. Second, it does not seem to be of tremendous importance, as our understanding of fractionally quantized Hall liquids is rather complete, and the number of questions we might wish to address at this stage is limited. To mention two possible applications, one could compare Jain's composite fermion quasi-electron (and hierarchy) wave functions to Laughlin's construction in a plane without boundaries. This would provide a complementary view to similar investigations on the sphere ${ }^{42}$, where the result reflects the different clustering properties of the Jack polynomials describing both states ${ }^{43}$. Furthermore, modular invariance arising in the context of the calculation of Hall viscosity ${ }^{44}$ might be investigated with the toroidal Laughlin quasi-particle wave functions at hand, and shed further light on similarities and differences to alternative approaches ${ }^{40}$.

Viewed from a broader perspective, however, it is best not to judge the importance of solutions to problems before we have obtained them. In solving a problem, and in 
solving a hard problem in particular, we may learn something which might turn out useful in a different context later on. With this in mind, we now revisit Laughlin's theory on the torus, and present a complete formulation.

The paper is organized as follows. In sections II and III, we review the ladder operator formalism for Landau level quantization, quasi-periodic boundary conditions, and single-particle states on the torus in symmetric gauge. Following Haldane and Rezayi ${ }^{34}$, we then construct Laughlin's wave functions for the ground states and quasi-hole excitations of fractionally quantized Hall liquids on the torus in sections IV and V. In section VI, we finally construct Laughlin's quasi-electron wave function to the torus. In the conclusion VII, we compare our result for the quasi-electron wave function to the quasihole wave function of Haldane and Rezayi, and speculate why the problem of constructing the quasi-electron has not been solved previously.

\section{LANDAU LEVELS, LADDER OPERATORS, AND MAGNETIC TRANSLATIONS}

To describe the dynamics of charged particles (e.g. spin-polarized electrons) in a two-dimensional plane subject to a perpendicular magnetic field $\boldsymbol{B}=-B \boldsymbol{e}_{\mathrm{z}}$, it is convenient to introduce complex particle coordinates $z=x+\mathrm{i} y$ and $\bar{z}=x-\mathrm{i} y^{45,46}$. The associated derivative operators are

$$
\partial_{z}=\frac{1}{2}\left(\partial_{x}-\mathrm{i} \partial_{y}\right), \partial_{\bar{z}}=\frac{1}{2}\left(\partial_{x}+\mathrm{i} \partial_{y}\right)
$$

Note that hermitian conjugation yields a - sign,

$$
\left(\partial_{z}\right)^{\dagger}=-\partial_{\bar{z}}
$$

The single particle Hamiltonian is obtained by minimally coupling the gauge field to the canonical momentum,

$$
H=\frac{1}{2 M}\left(\boldsymbol{p}+\frac{e}{c} \boldsymbol{A}\right)^{2},
$$

where $M$ is the mass of the particle and $e>0$. For our purposes, and in particular for the formulation of the quasi-electron excitations on the torus, it is more convenient to work in symmetric gauge $\boldsymbol{A}=\frac{1}{2} B \boldsymbol{r} \times \boldsymbol{e}_{\mathrm{z}}$, rather than in the Landau gauge used by Haldane and Rezayi ${ }^{34}$.

With the definition of the magnetic length $l=\sqrt{\hbar c / e B}$ and the ladder operators ${ }^{46-48,49}$ describing the cyclotron variables

$$
a=\sqrt{2} l\left(\partial_{\bar{z}}+\frac{1}{4 l^{2}} z\right), a^{\dagger}=\sqrt{2} l\left(-\partial_{z}+\frac{1}{4 l^{2}} \bar{z}\right),
$$

which obey $\left[a, a^{\dagger}\right]=1$, we may rewrite (3) as

$$
H=\hbar \omega_{\mathrm{c}}\left(a^{\dagger} a+\frac{1}{2}\right)
$$

where $\omega_{\mathrm{c}}=e B / M c$ is the cyclotron frequency. The kinetic energy of charged particles in a perpendicular magnetic field is hence quantized like a harmonic oscillator (Landau level quantization). The lowest Landau level (LLL) consists of those states annihilated by $a$. We introduce a second set of ladder operators describing the guiding center variables,

$$
b=\sqrt{2} l\left(\partial_{z}+\frac{1}{4 l^{2}} \bar{z}\right), b^{\dagger}=\sqrt{2} l\left(-\partial_{\bar{z}}+\frac{1}{4 l^{2}} z\right) .
$$

They likewise obey $\left[b, b^{\dagger}\right]=1$ and commute with the cyclotron ladder operators,

$$
[a, b]=\left[a, b^{\dagger}\right]=0 .
$$

For our purposes, it will be convenient to write the ladder operators as

$$
\begin{aligned}
(a, b) & =\sqrt{2} l e^{-S}\left(\partial_{\bar{z}}, \partial_{z}\right) e^{+S} \\
\left(a^{\dagger}, b^{\dagger}\right) & =\sqrt{2} l e^{-S}\left(-\partial_{z}+\frac{1}{2 l^{2}} \bar{z},-\partial_{\bar{z}}+\frac{1}{2 l^{2}} z\right) e^{+S},
\end{aligned}
$$

where $S \equiv|z|^{2} / 4 l^{2}$. Since the canonical angular momentum

$$
\boldsymbol{L}=\boldsymbol{r} \times \boldsymbol{p}=\hbar\left(b^{\dagger} b-a^{\dagger} a\right) \boldsymbol{e}_{\mathrm{z}}
$$

commutes with the Hamiltonian (5), we can use it to classify the vastly degenerate states within each Landau level. A complete, orthonormal set of basis states is

$$
\phi_{n, m}(z)=\frac{1}{\sqrt{n !}} \frac{1}{\sqrt{m !}}\left(a^{\dagger}\right)^{n}\left(b^{\dagger}\right)^{m} \frac{1}{\sqrt{2 \pi} l} e^{-S},
$$

where we omitted $\bar{z}$ from the argument of the wave functions as a choice of convention. Here $n+1$ denotes the Landau level index, and $m-n$ the angular momentum around the origin. With the particle position given by $z=\sqrt{2} l\left(a+b^{\dagger}\right)$, we find for the states (11) in the LLL

$$
\phi_{0, m}(z)=\frac{1}{\sqrt{2^{m+1} \pi m !} l^{m+1}} z^{m} e^{-S} .
$$

These states is describe narrow rings centered around the origin, with the radius determined by

$$
\left.\frac{\partial}{\partial r}\left|\phi_{0, m}(r)\right|^{2}\right|_{r=r_{m}} \stackrel{!}{=} 0
$$

which yields $r_{m}=\sqrt{2 m} l$. Since there are also $m$ states inside the ring, the areal degeneracy is

$$
\frac{\text { number of states }}{\text { area }}=\frac{m}{\pi r_{m}^{2}}=\frac{1}{2 \pi l^{2}},
$$

The magnetic flux required for each state,

$$
2 \pi l^{2} B=\frac{2 \pi \hbar c}{e}=\Phi_{0},
$$


is hence given by the Dirac flux quantum.

We can write the most general single particle state in the LLL as

$$
\psi(z)=e^{-S} g(z),
$$

where $g(z)$ is an analytic function of $z$.

Since the Hamiltonian (5) does not commute with translations, but only with translations supplemented by gauge transformations, we introduce the magnetic translation operator

$$
t(\xi) \equiv \exp \left(\frac{1}{\sqrt{2} l}\left(\xi b-\bar{\xi} b^{\dagger}\right)\right) .
$$

It obviously commutes with (5), but Baker-Hausdorff,

$$
e^{x+y}=e^{x} e^{y} e^{-\frac{1}{2}[x, y]} \text { for }[[x, y], x]=[[x, y], y]=0,
$$

implies

$$
t\left(\xi_{1}\right) t\left(\xi_{2}\right)=t\left(\xi_{1}+\xi_{2}\right) \exp \left(\frac{1}{4 l^{2}}\left(\bar{\xi}_{1} \xi_{2}-\xi_{1} \bar{\xi}_{2}\right)\right),
$$

and $\bar{\xi}_{1} \xi_{2}-\xi_{1} \bar{\xi}_{2}=2 \mathrm{i}\left(\boldsymbol{r}_{1} \times \boldsymbol{r}_{2}\right)_{\mathrm{z}}$, magnetic translations along different directions $t\left(\xi_{1}\right)$ and $t\left(\xi_{2}\right)$ commute only if the area spanned by $\xi_{1}$ and $\xi_{2}$ in the plane contains an integer number $N_{\phi}$ of magnetic Dirac flux quanta, i.e., $\left(\boldsymbol{r}_{1} \times \boldsymbol{r}_{2}\right)_{\mathrm{z}}=2 \pi l^{2} N_{\phi}$. This condition reflects Dirac's quantization condition for magnetic monopoles, which implies that the magnetic flux through any closed surface is given by an integer number of Dirac flux quanta.

\section{SINGLE PARTICLE STATES ON THE TORUS}

We impose periodic boundary conditions (PBCs) by

$$
t\left(\xi_{\alpha}\right) \psi(z)=e^{\mathrm{i} \phi_{\alpha}} \psi(z), \quad \text { for } \alpha=1, \tau,
$$

where $\xi_{1}$ and $\xi_{\tau}$ are two nonparallel displacements in the complex plane, and $\phi_{1}$ and $\phi_{\tau}$ are boundary phases. Since the boundary conditions require that $t\left(\xi_{1}\right)$ and $t\left(\xi_{\tau}\right)$ commute, the parallelogram spanned by $\xi_{1}$ and $\xi_{\tau}$ must contain an integer number of flux quanta, which we call $N_{\phi}$.

Due of the magnetic field, the wave functions subject to (17) are not strictly periodic, but only quasi-periodic,

$$
\psi\left(z+\xi_{\alpha}\right)=\exp \left(-\frac{1}{4 l^{2}}\left(\xi_{\alpha} \bar{z}-\bar{\xi}_{\alpha} z\right)\right) e^{\mathrm{i} \phi_{\alpha}} \psi(z) .
$$

We set the principal displacements $\xi_{1}=1$ and $\xi_{\tau}=\tau$, with $\operatorname{Im}(\tau)>0$, and call the region bounded by the four points $z=\frac{1}{2}( \pm 1 \pm \tau)$ the principal region. This fixes the magnetic length according to

$$
2 \pi l^{2} N_{\phi}=\operatorname{Im}(\tau) .
$$

For our purposes, it is most convenient to write the eigenstates as

$$
\psi(z)=e^{-S} f(z) e^{z^{2} / 4 l^{2}}
$$

These states are related by a simple gauge transformation from Landau to symmetric gauge to the eigenstates used by Haldane and Rezayi ${ }^{34}$ (see Eqn. (1) of their paper). It is further convenient to introduce the operator

$$
\begin{aligned}
\tilde{t}(\xi) & \equiv e^{+S} t(\xi) e^{-S} \\
& =\exp \left(\xi \partial_{z}+\bar{\xi} \partial_{\bar{z}}-\frac{1}{2 l^{2}} \bar{\xi} z\right) \\
& =\exp \left(-\frac{1}{4 l^{2}} \bar{\xi}(2 z+\xi)\right) \exp \left(\xi \partial_{z}\right)
\end{aligned}
$$

where we omitted the term involving $\partial_{\bar{z}}$. (This is possible because $\tilde{t}(\xi)$ acts throughout this article only on a function of $z$, not $\bar{z}$.) With

$$
\begin{aligned}
& \tilde{t}(1) e^{z^{2} / 4 l^{2}}=e^{z^{2} / 4 l^{2}}, \\
& \tilde{t}(\tau) e^{z^{2} / 4 l^{2}}=e^{z^{2} / 4 l^{2}} e^{i \pi N_{\phi}(2 z+\tau)},
\end{aligned}
$$

the PBCs (17) for $\psi(z)$ as specified by (20) imply

$$
\begin{aligned}
& \frac{f(z+1)}{f(z)}=e^{\mathrm{i} \phi_{1}}, \\
& \frac{f(z+\tau)}{f(z)}=e^{-\mathrm{i} \pi N_{\phi}(2 z+\tau)} e^{\mathrm{i} \phi_{\tau}} .
\end{aligned}
$$

As Haldane and Rezayi ${ }^{34}$ have pointed out, (24) and (25) can be used to calculate the numbers of zeros of $f(z)$ in the principal region (PR). With the theorem of residues, we obtain

$$
\begin{aligned}
& \frac{1}{2 \pi \mathrm{i}} \oint_{\mathrm{PR}} d z \frac{f^{\prime}(z)}{f(z)}=\frac{1}{2 \pi \mathrm{i}} \oint_{\mathrm{PR}} d(\ln f(z)) \\
& =\frac{1}{2 \pi \mathrm{i}}\left[\int_{-\frac{1}{2}+\frac{\tau}{2}}^{-\frac{1}{2}-\frac{\tau}{2}} d\left[\ln \frac{f(z)}{f(z+1)}\right]+\int_{-\frac{1}{2}-\frac{\tau}{2}}^{\frac{1}{2}-\frac{\tau}{2}} d\left[\ln \frac{f(z)}{f(z+\tau)}\right]\right] \\
& =\frac{1}{2 \pi \mathrm{i}} \int_{-\frac{1}{2}-\frac{\tau}{2}}^{\frac{1}{2}-\frac{\tau}{2}} d\left(i \pi N_{\phi}(2 z+\tau)-\mathrm{i} \phi_{\tau}\right)=N_{\phi}
\end{aligned}
$$

The function $f(z)$, and hence also the wave function $\psi(z)$, has hence as many zeros as there are Dirac flux quanta in the principal region.

The most general form for $f(z)$ is hence

$$
f(z)=e^{\mathrm{i} k z} \prod_{\nu=1}^{N_{\phi}} \vartheta_{\frac{1}{2}, \frac{1}{2}}\left(z-z_{\nu} \mid \tau\right),
$$

where $k$ is a real parameter $|k| \leq \pi N_{\phi}$, all the zeros $z_{\nu}$ are located in the principal region, and $\vartheta_{\frac{1}{2}, \frac{1}{2}}(z \mid \tau)$ is the odd 
Jacobi theta function ${ }^{50}$. The theta functions are defined in general for $a, b=0, \frac{1}{2}$ by

$$
\vartheta_{a, b}(z \mid \tau)=\sum_{n=-\infty}^{+\infty} e^{\pi \mathrm{i}(n+a)^{2} \tau} e^{2 \pi \mathrm{i}(n+a)(z+b)}
$$

and satisfy the quasi-periodicity relations

$$
\begin{aligned}
& \vartheta_{a, b}(z+1 \mid \tau)=e^{2 \pi \mathrm{i} a} \vartheta_{a, b}(z \mid \tau), \\
& \vartheta_{a, b}(z+\tau \mid \tau)=e^{-\pi \mathrm{i} \tau} e^{-2 \pi \mathrm{i}(z+b)} \vartheta_{a, b}(z \mid \tau) .
\end{aligned}
$$

The latter formula implies

$$
\vartheta_{a, b}(z+n \tau \mid \tau)=e^{-\pi \mathrm{i} \tau n^{2}} e^{-2 \pi \mathrm{i} n(z+b)} \vartheta_{a, b}(z \mid \tau) .
$$

Substitution of (27) into (24) and (25) yields that $k$ and the zeros $z_{\nu}$ are subject to the boundary conditions

$$
\begin{aligned}
& (-1)^{N_{\phi}} \exp (\mathrm{i} k)=e^{\mathrm{i} \phi_{1}}, \\
& (-1)^{N_{\phi}} \exp (\mathrm{i} k \tau) \exp \left(2 \pi \mathrm{i} \sum_{\nu=1}^{N_{\phi}} z_{\nu}\right)=e^{\mathrm{i} \phi_{\tau}} .
\end{aligned}
$$

For fixed values of the boundary phases $\phi_{1}$ and $\phi_{\tau}$, (32) and (33) possess a total of $N_{\phi}^{2}$ solutions for $k$ and $\sum_{\nu} z_{\nu}$; no further restrictions for the allowed choices of the individual $z_{\nu}$ result. Of all these distinct solutions, however, only $N_{\phi}$ yield linearly independent states. This is physically obvious, as there are only $N_{\phi}$ independent states in the LLL. It can also be seen from a simple mathematical consideration, as we will elaborate now.

The abstract property we require of $f(z)$ is to be analytic in $z$ and have exactly $N_{\phi}$ zeros in the principal region. Given one such solution $f(z)$, its ratio $\tilde{f}(z) / f(z)$ with any other solution $\tilde{f}(z)$ is a meromorphic truly periodic function on the torus, with at most simple poles at $N_{\phi}$ prescribed points (namely the zeroes of $f(z)$ ). It is a general theorem of complex function theory (a special case of the Riemann-Roch theorem) that the space of such functions is $N_{\phi}$ dimensional, including the constant function ${ }^{51}$. Hence there are only $N_{\phi}$ independent states.

\section{LAUGHLIN'S GROUND STATES}

Generalizing the formalism developed in the previous section, we write the most general $N$ particle state in the LLL as

$$
\psi[z]=e^{-S} f[z] \prod_{i=1}^{N} e^{z_{i}^{2} / 4 l^{2}},
$$

where $[z] \equiv\left(z_{1}, z_{2}, \ldots, z_{N}\right)$,

$$
e^{-S}=\prod_{i=1}^{N} e^{-\left|z_{i}\right|^{2} / 4 l^{2}},
$$

and $f\left(z_{1}, z_{2}, \ldots, z_{N}\right)$ is a completely antisymmetric (symmetric) function of the $z_{i}$ 's for fermions (bosons). It is subject to the PBCs

$$
t_{i}\left(\xi_{\alpha}\right) \psi[z]=e^{\mathrm{i} \phi_{\alpha}} \psi[z], \quad \text { for all } i, \alpha=1, \tau,
$$

where

$$
t_{i}(\xi) \equiv \exp \left(\frac{1}{\sqrt{2} l}\left(\xi b_{i}-\bar{\xi} b_{i}^{\dagger}\right)\right) .
$$

effects a magnetic translation of particle $z_{i}$. As in the previous section, the PBCs imply

$$
\begin{aligned}
& \frac{f\left(z_{1}+1, z_{2}, \ldots, z_{N}\right)}{f[z]}=e^{\mathrm{i} \phi_{1}}, \\
& \frac{f\left(z_{1}+\tau, z_{2}, \ldots, z_{N}\right)}{f[z]}=e^{-\mathrm{i} \pi N_{\phi}\left(2 z_{1}+\tau\right)} e^{\mathrm{i} \phi_{\tau}} .
\end{aligned}
$$

The characteristic feature of Laughlin's Jastrow-type states at Landau level filling fraction $\nu=1 / m$ is that a test particle $z_{1}$ sees exactly $m$ zeros at the locations of all the other particles $z_{2}, \ldots, z_{N}$. With $N_{\phi}=m N$, this requirement fixes the location of all but $m$ zeros in each of the coordinates. Following Haldane and Rezayi ${ }^{34}$, we are led to try an Ansatz

$$
f[z]=F(Z) \prod_{i<j}^{N} \vartheta_{\frac{1}{2}, \frac{1}{2}}\left(z_{i}-z_{j} \mid \tau\right)^{m},
$$

where

$$
Z \equiv \sum_{i=1}^{N} z_{i}
$$

is the center-of-mass coordinate. Substitution of (40) into (38) and (39) yields

$$
\begin{aligned}
& \frac{F(Z+1)}{F(Z)}=(-1)^{N_{\phi}-m} e^{\mathrm{i} \phi_{1}}, \\
& \frac{F(Z+\tau)}{F(Z)}=(-1)^{N_{\phi}-m} e^{-\mathrm{i} \pi m(2 Z+\tau)} e^{\mathrm{i} \phi_{\tau}} .
\end{aligned}
$$

These equations are extremely similar to (24) and (25), and imply via

$$
\frac{1}{2 \pi \mathrm{i}} \oint_{\mathrm{PR}} d(\ln F(Z))=m
$$

that $F(Z)$ has exactly $m$ zeros in the principal region.

In analogy to (27), the most general solution for the single particle states

$$
F(Z)=e^{\mathrm{i} K Z} \prod_{\nu=1}^{m} \vartheta_{\frac{1}{2}, \frac{1}{2}}\left(Z-Z_{\nu} \mid \tau\right)
$$

where $K$ is a real parameter $|K| \leq \pi m$, and all the zeros $Z_{\nu}$ are located in the principal region. Substitution of 
(45) into (42) and (43) yields that $K$ and the center-ofmass zeros $Z_{\nu}$ are subject to the boundary conditions

$$
\begin{aligned}
& (-1)^{N_{\phi}} \exp (\mathrm{i} K)=e^{\mathrm{i} \phi_{1}}, \\
& (-1)^{N_{\phi}} \exp (\mathrm{i} K \tau) \exp \left(2 \pi \mathrm{i} \sum_{\nu=1}^{m} Z_{\nu}\right)=e^{\mathrm{i} \phi_{\tau}} .
\end{aligned}
$$

For fixed values of the boundary phases $\phi_{1}$ and $\phi_{\tau},(46)$ and (47) possess a total of $m^{2}$ solutions for $k$ and $\sum_{\nu} z_{\nu}$. As above, only $m$ of these solutions yield linearly independent states. We can see this either from the equivalence of the problem to (32) and (33), or directly from the mathematical argument indicated there.

The $m$-fold degeneracy of the Laughlin $1 / m$ state on a closed surface with genus one is a topological quantum number which characterizes the topological order of the state.

\section{QUASI-HOLE EXCITATIONS}

The generalization of this formalism to include a quasihole excitation at position $\eta$ in the complex plane follows without incident. While in many applications it is sufficient to view $\eta$ as a parameter, there are some which require to view it as a dynamical variable. An example of an application of the latter type is the hierarchy of quantized Hall states, where the quasi-particle excitations themselves condense into Laughlin-Jastrow-type fluids ${ }^{2-4}$.

We hence set up the formalism as general as possible, and write the wave function with one quasi-hole as

$$
\psi_{\eta}[z]=e^{-S} f_{\eta}[z] e^{\eta^{2} / 4 m l^{2}} \prod_{i=1}^{N} e^{z_{i}^{2} / 4 l^{2}},
$$

where

$$
e^{-S}=e^{-|\eta|^{2} / 4 m l^{2}} \prod_{i=1}^{N} e^{-\left|z_{i}\right|^{2} / 4 l^{2}},
$$

and $N_{\phi}=m N+1$. Since the quasi-hole carries a fraction of $1 / m$ of an electron charge, the effective magnetic length for the quasi-holes is $\sqrt{m}$ times the magnetic length $l$ seen by the electrons. In analogy to the ground state, the PBCs (36) for (48) imply the conditions (38) and (39) for $f_{\eta}[z]$.

Since the quasi-hole excitation amounts to an isolated zero seen by all the particles, we consider an Ansatz

$$
f_{\eta}[z]=F_{\eta}(Z) \prod_{i=1}^{N} \vartheta_{\frac{1}{2}, \frac{1}{2}}\left(z_{i}-\eta \mid \tau\right) \prod_{i<j}^{N} \vartheta_{\frac{1}{2}, \frac{1}{2}}\left(z_{i}-z_{j} \mid \tau\right)^{m},
$$

where the center-of-mass coordinate now includes the quasi-hole,

$$
F_{\eta}(Z)=e^{\mathrm{i} K(Z+\eta / m)} \prod_{\nu=1}^{m} \vartheta_{\frac{1}{2}, \frac{1}{2}}\left(Z+\frac{\eta}{m}-Z_{\nu} \mid \tau\right),
$$

With $Z$ once again given by (41), we find that (38) and (39) for $f_{\eta}[z]$ are satisfied if $K$ and the center-of-mass ze$\operatorname{ros} Z_{\nu}$ are once again subject to the boundary conditions (46) and (47).

Regarding the quasi-periodicity in the quasi-hole coordinate $\eta$, we begin by introducing ladder operators for the quasi-hole coordinate,

$$
\begin{aligned}
c & =\sqrt{2 m l} l e^{-S} \partial_{\eta} e^{+S}, \\
c^{\dagger} & =\sqrt{2 m l} l e^{-S}\left(-\partial_{\bar{\eta}}+\frac{1}{2 m l^{2}} \eta\right) e^{+S},
\end{aligned}
$$

where $S$ is given by (49). (Note that for the purposes of defining the operators, we could have just as well taken $S=|\eta|^{2} / 4 m l^{2}$, as the terms depending on the $z_{i}$ 's cancel in (52) and (53).) $c$ and $c^{\dagger}$ obey the commutation relation $\left[c, c^{\dagger}\right]=1$ and trivially commute with $a, a^{\dagger}, b$, and $b^{\dagger}$. We define the magnetic translation operator for the quasi-hole coordinate

$$
t_{\eta}(\xi) \equiv \exp \left(\frac{1}{\sqrt{2 m l}}\left(\xi c-\bar{\xi} c^{\dagger}\right)\right) .
$$

Since

$$
t_{\eta}\left(\xi_{1}\right) t_{\eta}\left(\xi_{2}\right)=t_{\eta}\left(\xi_{1}+\xi_{2}\right) \exp \left(\frac{1}{4 m l^{2}}\left(\bar{\xi}_{1} \xi_{2}-\xi_{1} \bar{\xi}_{2}\right)\right),
$$

magnetic quasi-hole translations along different directions $t_{\eta}\left(\xi_{1}\right)$ and $t_{\eta}\left(\xi_{2}\right)$ commute only if the area spanned by $\xi_{1}$ and $\xi_{2}$ in the plane contains $m$ times an integer number $N_{\phi}$ of magnetic Dirac flux quanta (or equivalently, an integer number of "quasi-hole flux quanta" $m \Phi_{0}$, where $\Phi_{0}$ is the Dirac flux quantum).

From both these consideration and (51), we conclude that in general, we do not expect the quasi-hole wave function to be quasi-periodic under translations by 1 and $\tau$, but only under translations by $m$ and $m \tau$. As a side note, we can achieve quasi-periodicity by quasihole translations of either 1 and $m \tau$ or $m$ and $\tau$, if we arrange the center-of-mass zeros with equal spacing along one of the meridians of the torus,

$$
Z_{\nu}=Z_{1}+\frac{(\nu-1)}{m} \quad \text { or } \quad Z_{\nu}=Z_{1}+\frac{(\nu-1) \tau}{m} .
$$

From (50) with (51), we can readily derive

$$
\begin{aligned}
\frac{f_{\eta+m}[z]}{f_{\eta}[z]}= & (-1)^{m(N+1)} e^{\mathrm{i} K} \\
\frac{f_{\eta+m \tau}[z]}{f_{\eta}[z]}= & (-1)^{m(N+1)} e^{\mathrm{i} K \tau} e^{-\mathrm{i} \pi N_{\phi}(2 \eta+m \tau)} \\
& \cdot \exp \left(2 \pi \mathrm{i} \sum_{\nu=1}^{m} Z_{\nu}\right)
\end{aligned}
$$


In analogy to our elaborations in Section III, we introduce an operator

$$
\begin{aligned}
\tilde{t}_{\eta}(\xi) & \equiv e^{+S} t_{\eta}(\xi) e^{-S} \\
& =\exp \left(\xi \partial_{\eta}+\bar{\xi} \partial_{\bar{\eta}}-\frac{1}{2 m l^{2}} \bar{\xi} \eta\right) \\
& =\exp \left(-\frac{1}{4 m l^{2}} \bar{\xi}(2 \eta+\xi)\right) \exp \left(\xi \partial_{\eta}\right),
\end{aligned}
$$

where we omitted the term involving $\partial_{\bar{\eta}}$ (knowing that it will only act on functions of $\eta$, not $\bar{\eta}$ ). With

$$
\begin{aligned}
\tilde{t}_{\eta}(m) e^{\eta^{2} / 4 m l^{2}} & =e^{\eta^{2} / 4 m l^{2}}, \\
\tilde{t}_{\eta}(m \tau) e^{\eta^{2} / 4 m l^{2}} & =e^{i \pi N_{\phi}(2 \eta+m \tau)} e^{\eta^{2} / 4 m l^{2}},
\end{aligned}
$$

we find that

$$
\begin{aligned}
t_{\eta}(m) \psi_{\eta}[z] & =(-1)^{N_{\phi}+m-1} e^{\mathrm{i} K} \psi_{\eta}[z] \\
t_{\eta}(m \tau) \psi_{\eta}[z] & =(-1)^{N_{\phi}+m-1} e^{\mathrm{i} K \tau} \exp \left(2 \pi \mathrm{i} \sum_{\nu=1}^{m} Z_{\nu}\right) \psi_{\eta}[z] .
\end{aligned}
$$

With the boundary conditions (46) and (47), this implies that

$$
t_{\eta}\left(m \xi_{\alpha}\right) \psi_{\eta}[z]=e^{\mathrm{i} \phi_{\alpha}}(-1)^{m-1} \psi_{\eta}[z], \quad \text { for } \alpha=1, \tau,
$$

with $\xi_{1}=1$ and $\xi_{\tau}=\tau$. For fermions, $m$ is odd and $(-1)^{m-1}=1$. As we (magnetically) translate the quasihole with charge $e^{*}=e / m m$ times around one of the meridians of the torus, the ground state wave function acquires the same phase as it acquires when we translate an electron with charge $-e$ once around.

The example of arranging the center-of-mass zeros according to (56) lends itself well to illustrate the connection between translations of the quasi-hole once around one of the meridians of the torus and the topological degeneracy of the Laughlin states on higher genus surfaces. As both cases mentioned above are analogous, we only elaborate the first one, where

$$
Z_{\nu}=Z_{1}+\frac{(\nu-1)}{m}
$$

If we (magnetically) translate the quasi-hole by 1 , the center-of-mass zeros transform according to

$$
Z_{\nu} \rightarrow Z_{\nu-1} \quad \text { for } \nu>1, \quad Z_{1} \rightarrow Z_{m}
$$

Magnetic translation of the quasi-hole by 1 will hence only yield a phase in the wave function,

$$
t_{\eta}(1) \psi_{\eta}[z]=(-1)^{N+1} e^{\mathrm{i} K / m} \psi_{\eta}[z] .
$$

If we magnetically translate the quasi-hole via $t_{\eta}(\tau)$ by $\tau$, however, all the center-of-mass zeros will by shifted,

$$
Z_{\nu} \rightarrow Z_{\nu}-\frac{\tau}{m} \text { for } \nu
$$

We hence obtain a different, topologically degenerate ground state. From the real part of boundary condition (47), we see that (64) implies $K \rightarrow K+2 \pi$, and hence via (63) that the new state is orthogonal to the original one. After $m$ magnetic translations by $\tau$, we finally recover the original state modulo a phase, as specified by (60).

Even though we might have clarified some details and increased the accessibility, all of the results presented so far have been understood by Haldane and Rezayi ${ }^{34}$, as well as the community at large. The wave functions for the quasi-electron excitations on the torus we derive in the following section, however, have to our knowledge not been obtained previously.

\section{QUASI-ELECTRON EXCITATIONS}

Before we dive into the details of how to construct Laughlin's quasi-electron wave function on the torus, let us briefly recall the construction in the plane. Laughlin obtains the quasi-hole by inserting one Dirac flux quantum adiabatically in the direction of the background magnetic field at a position $\eta$. In the process, all the electrons in the liquid acquire a shift of $\hbar$ in the canonical angular momentum around $\eta$, and the ground state evolves into

$$
\psi_{\eta}[z]=\prod_{i=1}^{N}\left(z_{i}-\eta\right) \prod_{i<j}^{N}\left(z_{i}-z_{j}\right)^{m} e^{-S} .
$$

As $m$ quasi-holes at $\eta$ would constitute a true hole with charge $+e$ in the liquid, the charge of the quasi-hole is $+e / m$.

The quasi-electron, i.e., the antiparticle of the quasihole, has charge $-e / m$ and is created by inserting the flux adiabatically in the opposite direction, thus lowering the angular momentum around some position $\eta$ by $\hbar$, or alternatively, by removing one of the zeros from the wave function. To accomplish this formally, we first rewrite (65) in terms of ladder operators:

$$
\psi_{\eta}[z]=\prod_{i=1}^{N}\left(\sqrt{2} l b_{i}^{\dagger}-\eta\right) \prod_{i<j}^{N}\left(z_{i}-z_{j}\right)^{m} e^{-S} .
$$

The insertion of a flux quantum in the opposite direction, or the lowering of the angular momentum around $\eta$, will then correspond to the Hermitian conjugate operation. Laughlin $^{31}$ hence proposed for the quasi-electron wave function in the plane ${ }^{52}$

$$
\begin{aligned}
\psi_{\bar{\eta}}[z] & =\prod_{i=1}^{N}\left(\sqrt{2} l b_{i}-\bar{\eta}\right) \prod_{i<j}^{N}\left(z_{i}-z_{j}\right)^{m} e^{-S} \\
& =e^{-S} \prod_{i=1}^{N}\left(2 l^{2} \partial_{z_{i}}-\bar{\eta}\right) \prod_{i<j}^{N}\left(z_{i}-z_{j}\right)^{m} .
\end{aligned}
$$


Conducting the same operation with the quasi-hole factors in (50) we obtain with (28),

$$
\left[\vartheta_{\frac{1}{2}, \frac{1}{2}}\left(\sqrt{2} l b_{i}^{\dagger}-\eta \mid \tau\right)\right]^{\dagger}=\vartheta_{\frac{1}{2}, \frac{1}{2}}\left(\sqrt{2} l b_{i}-\bar{\eta} \mid-\bar{\tau}\right) .
$$

These considerations suggest that for the wave function with a quasi-electron at $\bar{\eta}$ on the torus, we may consider an Ansatz of the form

$$
\psi_{\bar{\eta}}[z]=e^{-S} f_{\bar{\eta}, \partial_{\bar{\eta}}}\left[z, \partial_{z}\right] e^{R}
$$

where $S$ is still given by (49), $f$ depends now on derivatives of the coordinates $z_{i}$ and $\bar{\eta}$ as well, $N_{\phi}=m N-1$, and we have defined

$$
e^{R} \equiv e^{\bar{\eta}^{2} / 4 m l^{2}} \prod_{i=1}^{N} e^{z_{i}^{2} / 4 l^{2}} .
$$

The challenge is now to identify the functions $f_{\bar{\eta}, \partial_{\bar{\eta}}}\left[z, \partial_{z}\right]$ such that $\psi_{\bar{\eta}}[z]$ is quasi-periodic under translations of any $z_{i}$ around either meridians of the torus, and of $\bar{\eta}$ under $m$ such translations in either direction.

Regarding the $z_{i}$ 's, the PBCs are still given by (36) with (37). Since $f_{\bar{\eta}, \partial_{\bar{\eta}}}\left[z, \partial_{z}\right]$ contains derivative operators as well, however, we have to replace (38) and (39) by

$$
\begin{aligned}
& \tilde{t}_{1}(1) f_{\bar{\eta}, \partial_{\bar{\eta}}}\left[z, \partial_{z}\right] \tilde{t}_{1}(1)^{-1} e^{R} \\
& =\tilde{t}_{1}(1) f_{\bar{\eta}, \partial_{\bar{\eta}}}\left[z, \partial_{z}\right] e^{R}=e^{\mathrm{i} \phi_{1}} f_{\bar{\eta}, \partial_{\bar{\eta}}}\left[z, \partial_{z}\right] e^{R}, \\
& \tilde{t}_{1}(\tau) f_{\bar{\eta}, \partial_{\bar{\eta}}}\left[z, \partial_{z}\right] \tilde{t}_{1}(\tau)^{-1} e^{\mathrm{i} \pi N_{\phi}\left(2 z_{1}+\tau\right)} e^{R} \\
& =\tilde{t}_{1}(\tau) f_{\bar{\eta}, \partial_{\bar{\eta}}}\left[z, \partial_{z}\right] e^{R}=e^{\mathrm{i} \phi_{\tau}} f_{\bar{\eta}, \partial_{\bar{\eta}}}\left[z, \partial_{z}\right] e^{R},
\end{aligned}
$$

where $\tilde{t}_{1}(\xi)$ is according to (21) given by

$$
\tilde{t}_{1}(\xi)=\exp \left(-\frac{1}{4 l^{2}} \bar{\xi}\left(2 z_{1}+\xi\right)\right) \exp \left(\xi \partial_{z_{1}}\right) .
$$

$\tilde{t}_{1}(\xi)$ translates both $z_{1} \rightarrow z_{1}+\xi$ and $2 l^{2} \partial_{z_{1}} \rightarrow 2 l^{2} \partial_{z_{1}}+\bar{\xi}$. (Some care is required because the operators effecting these actions do not commute.)

Regarding the quasi-periodicity in the quasi-electron coordinate $\bar{\eta}$, we introduce yet another set of ladder operators for the quasi-electron coordinate,

$$
\begin{aligned}
\bar{c} & =\sqrt{2 m l} e^{-S} \partial_{\bar{\eta}} e^{+S}, \\
\bar{c}^{\dagger} & =\sqrt{2 m} l e^{-S}\left(-\partial_{\eta}+\frac{1}{2 m l^{2}} \bar{\eta}\right) e^{+S},
\end{aligned}
$$

where $S$ is still given by (49). (Again, for the purposes of defining the operators, we could have just as well taken $S=|\eta|^{2} / 4 m l^{2}$, as the terms depending on the $z_{i}$ 's cancel in (74) and (75).) $\bar{c}$ and $\bar{c}^{\dagger}$ obey the commutation relation $\left[\bar{c}, \bar{c}^{\dagger}\right]=1$ and trivially commute with $a, a^{\dagger}, b$, and $b^{\dagger}$. We define the magnetic translation operator for the quasi-electron coordinate,

$$
t_{\bar{\eta}}(\xi) \equiv \exp \left(\frac{1}{\sqrt{2 m} l}\left(\bar{\xi} \bar{c}-\xi \bar{c}^{\dagger}\right)\right) .
$$

As for the quasi-hole, $t_{\bar{\eta}}\left(\xi_{1}\right)$ and $t_{\bar{\eta}}\left(\xi_{2}\right)$ commute only if the area spanned by $\xi_{1}$ and $\xi_{2}$ in the plane contains $m$ times an integer number $N_{\phi}$ of magnetic Dirac flux quanta.

As in the previous sections, we further introduce an operator

$$
\begin{aligned}
\tilde{t}_{\bar{\eta}}(\xi) & \equiv e^{+S} t_{\bar{\eta}}(\xi) e^{-S} \\
& =\exp \left(\bar{\xi} \partial_{\bar{\eta}}+\xi \partial_{\eta}-\frac{1}{2 m l^{2}} \xi \bar{\eta}\right) \\
& =\exp \left(-\frac{1}{4 m l^{2}} \xi(2 \bar{\eta}+\bar{\xi})\right) \exp \left(\bar{\xi} \partial_{\bar{\eta}}\right)
\end{aligned}
$$

where we again omitted the term involving $\partial_{\eta}$ (knowing that it will only act on functions of $\bar{\eta}$, not $\eta$ ). Acting on the factor $e^{R}$ to the left of $f_{\bar{\eta}, \partial_{\bar{\eta}}}\left[z, \partial_{z}\right]$ in (69) yields

$$
\begin{aligned}
\tilde{t}_{\bar{\eta}}(m) e^{\bar{\eta}^{2} / 4 m l^{2}} & =e^{\bar{\eta}^{2} / 4 m l^{2}}, \\
\tilde{t}_{\bar{\eta}}(m \tau) e^{\bar{\eta}^{2} / 4 m l^{2}} & =e^{-i \pi N_{\phi}(2 \bar{\eta}+m \bar{\tau})} e^{\bar{\eta}^{2} / 4 m l^{2}} .
\end{aligned}
$$

In analogy to (60), we expect PBCs for the quasi-electron according to

$$
t_{\bar{\eta}}\left(m \xi_{\alpha}\right) \psi_{\bar{\eta}}[z]=e^{-\mathrm{i} \phi_{\alpha}}(-1)^{m-1} \psi_{\bar{\eta}}[z], \text { for } \alpha=1, \tau,
$$

where $\xi_{1}=1, \xi_{\tau}=\tau$. Note that we have reversed the signs of the boundary phases $\phi_{1}$ and $\phi_{\tau}$ as compared to the quasi-hole, accounting for the quasi-electron being the quasi-holes antiparticle. These boundary conditions translate into the following conditions for $f_{\bar{\eta}, \partial_{\bar{\eta}}}\left[z, \partial_{z}\right]$,

$$
\begin{aligned}
& \tilde{t}_{\bar{\eta}}(m) f_{\bar{\eta}, \partial_{\bar{\eta}}}\left[z, \partial_{z}\right] \tilde{t}_{\bar{\eta}}(m)^{-1} e^{R} \\
& =\tilde{t}_{\bar{\eta}}(m) f_{\bar{\eta}, \partial_{\bar{\eta}}}\left[z, \partial_{z}\right] e^{R} \\
& =e^{-\mathrm{i} \phi_{1}}(-1)^{m-1} f_{\bar{\eta}, \partial_{\bar{\eta}}}\left[z, \partial_{z}\right] e^{R} \text {, } \\
& \tilde{t}_{\bar{\eta}}(m \tau) f_{\bar{\eta}, \partial_{\bar{\eta}}}\left[z, \partial_{z}\right] \tilde{t}_{\bar{\eta}}(m \tau)^{-1} e^{-\mathrm{i} \pi N_{\phi}(2 \bar{\eta}+m \bar{\tau})} e^{R} \\
& =\tilde{t}_{\bar{\eta}}(m \tau) f_{\bar{\eta}, \partial_{\bar{\eta}}}\left[z, \partial_{z}\right] e^{R} \\
& =e^{-\mathrm{i} \phi_{\tau}}(-1)^{m-1} f_{\bar{\eta}, \partial_{\bar{\eta}}}\left[z, \partial_{z}\right] e^{R} \text {. }
\end{aligned}
$$

The challenge to identify the quasi-electron wave function is now reduced to constructing a quasi-electron (wave) function $f_{\bar{\eta}, \partial_{\bar{\eta}}}\left[z, \partial_{z}\right]$ with transformation properties $(71)$, (72) and $(81),(82)$ with $\tilde{t}_{1}(\xi)$ and $\tilde{t}_{\bar{\eta}}(\xi)$ given by (73) and (77), respectively.

From $(50),(68),(45)$, and (51), we expect $f_{\bar{\eta}, \partial_{\bar{\eta}}}\left[z, \partial_{z}\right]$ to contain factors

$$
\begin{aligned}
Q_{\bar{\eta}}\left[\partial_{z}\right] & =\prod_{i=1}^{N} \vartheta_{\frac{1}{2}, \frac{1}{2}}\left(2 l^{2} \partial_{z_{i}}-\bar{\eta} \mid-\bar{\tau}\right), \\
J[z] & =\prod_{i<j}^{N} \vartheta_{\frac{1}{2}, \frac{1}{2}}\left(z_{i}-z_{j} \mid \tau\right)^{m}
\end{aligned}
$$


and

$$
\begin{aligned}
F_{\partial_{\bar{\eta}}}(Z) & =\exp \left(\mathrm{i} K\left(Z-2 l^{2} \partial_{\bar{\eta}}\right)\right) \\
& \cdot \prod_{\nu=1}^{m} \vartheta_{\frac{1}{2}, \frac{1}{2}}\left(Z-2 l^{2} \partial_{\bar{\eta}}-Z_{\nu} \mid \tau\right),
\end{aligned}
$$

where $K$ is a real parameter $|K| \leq \pi m$, the center-ofmass coordinate now includes the quasi-electron coordinate in form of a partial derivative, and all the zeros $Z_{\nu}$ are located in the principal region. (The derivative in $\bar{\eta}$ in (85) is required because $\tilde{t}_{\bar{\eta}}(\xi)$ transforms $\bar{\eta} \rightarrow \bar{\eta}+\bar{\xi}$ but $2 m l^{2} \partial_{\bar{\eta}} \rightarrow 2 m l^{2} \partial_{\bar{\eta}}+\xi$.)

Since the transformation properties of these factors under translations of $z_{1}$ by 1 or $\bar{\eta}$ by $m$ are rather trivial, we proceed by investigating translations of $z_{1}$ by $\tau$ or $\bar{\eta}$ by $m \bar{\tau}$.

Explicit evaluation of the action of $\tilde{t}_{1}(\tau)$ on (83), (85), and (84) yields

$$
\begin{aligned}
& \tilde{t}_{1}(\tau) Q_{\bar{\eta}}\left[\partial_{z}\right] \tilde{t}_{1}(\tau)^{-1} \\
& =Q_{\bar{\eta}}\left[\partial_{z}\right](-1) e^{\mathrm{i} \pi \bar{\tau}} \exp \left(2 \pi \mathrm{i}\left(2 l^{2} \partial_{z_{1}}-\bar{\eta}\right)\right), \\
& \tilde{t}_{1}(\tau) F_{\partial_{\bar{\eta}}}(Z) \tilde{t}_{1}(\tau)^{-1} \\
& =F_{\partial_{\bar{\eta}}}(Z)(-1)^{m} e^{\mathrm{i} K \tau} e^{-\mathrm{i} \pi \tau m} \\
& \quad \cdot \exp \left(-2 \pi \mathrm{i}\left[m Z-2 m l^{2} \partial_{\bar{\eta}}-\sum_{\nu=1}^{m} Z_{\nu}\right]\right), \\
& \tilde{t}_{1}(\tau) J[z] \tilde{t}_{1}(\tau)^{-1} \\
& =J[z](-1)^{m(N-1)} e^{-\mathrm{i} \pi \tau m(N-1)} e^{-2 \pi \mathrm{i} m\left(N z_{1}-Z\right) .}
\end{aligned}
$$

While there are the usual cancelations between the terms on the right in (87) and (88), there is a complete mismatch with the terms in (86). To match them, we need to generate an additional factor

$\exp \left(-2 \pi \mathrm{i}\left(2 l^{2} \partial_{z_{1}}-\bar{\eta}\right)\right) \exp \left(2 \pi \mathrm{i}\left(z_{1}-2 m l^{2} \partial_{\bar{\eta}}\right)\right) e^{\mathrm{i} \pi(\tau-\bar{\tau})}$

between (86) and (87) under translation by $\tilde{t}_{1}(\tau)$. To this end, we introduce the Gaussian

$$
G_{\bar{\eta}, \partial_{\bar{\eta}}}\left[z, \partial_{z}\right]=\prod_{i=1}^{N} \exp \left(\frac{1}{4 m l^{2} N} D_{i}^{2}\right),
$$

where

$$
D_{i} \equiv z_{i}-2 l^{2} \partial_{z_{i}}+\bar{\eta}-2 m l^{2} \partial_{\bar{\eta}} .
$$

The transformation properties of $G_{\bar{\eta}, \partial_{\bar{\eta}}}\left[z, \partial_{z}\right]$ are (see Ap- pendix)

$$
\begin{aligned}
& \tilde{t}_{1}(1) G_{\bar{\eta}, \partial_{\bar{\eta}}}\left[z, \partial_{z}\right] \tilde{t}_{1}(1)^{-1}=G_{\bar{\eta}, \partial_{\bar{\eta}}}\left[z, \partial_{z}\right], \\
& \tilde{t}_{1}(\tau) G_{\bar{\eta}, \partial_{\bar{\eta}}}\left[z, \partial_{z}\right] \tilde{t}_{1}(\tau)^{-1} \\
& =\exp \left(-2 \pi \mathrm{i}\left(2 l^{2} \partial_{z_{1}}-\bar{\eta}\right)\right) \\
& \cdot G_{\bar{\eta}, \partial_{\bar{\eta}}}\left[z, \partial_{z}\right] \exp \left(-\frac{2 \pi \mathrm{i}}{N} D\right) \\
& \cdot \exp \left(2 \pi \mathrm{i}\left(z_{1}-2 m l^{2} \partial_{\bar{\eta}}\right)\right) e^{\mathrm{i} \pi(\tau-\bar{\tau})},
\end{aligned}
$$

where

$$
D \equiv \sum_{i=1}^{N} D_{i}
$$

Combining these properties, we find that

$$
f_{\bar{\eta}, \partial_{\bar{\eta}}}\left[z, \partial_{z}\right]=Q_{\bar{\eta}}\left[\partial_{z}\right] G_{\bar{\eta}, \partial_{\bar{\eta}}}\left[z, \partial_{z}\right] F_{\partial_{\bar{\eta}}}(Z) J[z]
$$

transforms as

$$
\begin{aligned}
\tilde{t}_{1}(\tau) f_{\bar{\eta}, \partial_{\bar{\eta}}}\left[z, \partial_{z}\right] \tilde{t}_{1}(\tau)^{-1} \\
=Q_{\bar{\eta}}\left[\partial_{z}\right] G_{\bar{\eta}, \partial_{\bar{\eta}}}\left[z, \partial_{z}\right] \exp \left(-\frac{2 \pi \mathrm{i}}{N} D\right) F_{\partial_{\bar{\eta}}}(Z) J[z] \\
\cdot(-1)^{N_{\phi}} e^{\mathrm{i} K \tau} \exp \left(2 \pi \mathrm{i} \sum_{\nu=1}^{m} Z_{\nu}\right) e^{-\mathrm{i} \pi N_{\phi}\left(2 z_{1}+\tau\right)}
\end{aligned}
$$

With

$$
\left[D, F_{\partial_{\bar{\eta}}}(Z)\right]=[D, J[z]]=0 \quad \text { and } \quad D_{i} e^{R}=0,
$$

we find with (95) that (94) satisfies (71) and (72) provided that the boundary conditions (46) and (47) are satisfied.

Under translations of the quasi-electron coordinate $\bar{\eta}$ by $m \bar{\tau}$ we find that the factors $Q_{\bar{\eta}}\left[\partial_{z}\right], F_{\partial_{\bar{\eta}}}(Z)$, and $J[z]$ transform as

$$
\begin{aligned}
& \tilde{t}_{\bar{\eta}}(m \tau) Q_{\bar{\eta}}\left[\partial_{z}\right] \tilde{t}_{\bar{\eta}}(m \tau)^{-1} \\
&= Q_{\bar{\eta}}\left[\partial_{z}\right](-1)^{m N} e^{\mathrm{i} \pi \bar{\tau} m^{2} N} \\
& \cdot \prod_{i=1}^{N} \exp \left(-2 \pi \mathrm{i} m\left(2 l^{2} \partial_{z_{i}}-\bar{\eta}\right)\right), \\
& \tilde{t}_{\bar{\eta}}(m \tau) F_{\partial_{\bar{\eta}}}(Z) \tilde{t}_{\bar{\eta}}(m \tau)^{-1} \\
&= F_{\partial_{\bar{\eta}}}(Z)(-1)^{m} e^{-\mathrm{i} K \tau} e^{-\mathrm{i} \pi \tau m} \\
& \cdot \exp \left(2 \pi \mathrm{i}\left[m Z-2 m l^{2} \partial_{\bar{\eta}}-\sum_{\nu=1}^{m} Z_{\nu}\right]\right), \\
& \tilde{t}_{\bar{\eta}}(m \tau) J[z] \tilde{t}_{\bar{\eta}}(m \tau)^{-1}=J[z],
\end{aligned}
$$


while the Gaussian factor transforms as (see Appendix)

$$
\begin{aligned}
\tilde{t}_{\bar{\eta}}(m) G_{\bar{\eta}, \partial_{\bar{\eta}}}\left[z, \partial_{z}\right] \tilde{t}_{\bar{\eta}}(m)^{-1}=G_{\bar{\eta}, \partial_{\bar{\eta}}}\left[z, \partial_{z}\right], \\
\tilde{t}_{\bar{\eta}}(m \tau) G_{\bar{\eta}, \partial_{\bar{\eta}}}\left[z, \partial_{z}\right] \tilde{t}_{\bar{\eta}}(m \tau)^{-1} \\
=\prod_{i=1}^{N} \exp \left(2 \pi \mathrm{i} m\left(2 l^{2} \partial_{z_{i}}-\bar{\eta}\right)\right) \\
\cdot G_{\bar{\eta}, \partial_{\bar{\eta}}}\left[z, \partial_{z}\right] \exp (2 \pi \mathrm{i} m D) \\
\cdot \prod_{i=1}^{N} \exp \left(-2 \pi \mathrm{i} m\left(z_{i}-2 m l^{2} \partial_{\bar{\eta}}\right)\right) e^{\mathrm{i} \pi m^{2} N(\tau-\bar{\tau})}
\end{aligned}
$$

Taken together, we obtain the transformation properties

$$
\begin{aligned}
\tilde{t}_{\bar{\eta}}(m \tau) & f_{\bar{\eta}, \partial_{\bar{\eta}}}\left[z, \partial_{z}\right] \tilde{t}_{\bar{\eta}}(m \tau)^{-1} \\
= & f_{\bar{\eta}, \partial_{\bar{\eta}}}\left[z, \partial_{z}\right] \\
& \cdot(-1)^{N_{\phi}} e^{-\mathrm{i} K \tau} \exp \left(-2 \pi \mathrm{i} \sum_{\nu=1}^{m} Z_{\nu}\right)(-1)^{m-1} \\
& \cdot \exp (2 \pi \mathrm{i} m D) e^{\mathrm{i} \pi \tau m N_{\phi}} \exp \left(2 \pi \mathrm{i} N_{\phi} 2 m l^{2} \partial_{\bar{\eta}}\right) .
\end{aligned}
$$

The last term generated in (102) does not resemble the term required by (82), but since

$$
\begin{aligned}
& e^{\mathrm{i} \pi \tau m N_{\phi}} \exp \left(2 \pi \mathrm{i} N_{\phi} 2 m l^{2} \partial_{\bar{\eta}}\right) e^{-i \pi N_{\phi}(2 \bar{\eta}+m \bar{\tau})} e^{\bar{\eta}^{2} / 4 m l^{2}} \\
& =\exp \left(m(\tau-\bar{\tau}) \partial_{\bar{\eta}}\right) e^{\bar{\eta}^{2} / 4 m l^{2}} e^{-i \pi N_{\phi}(2 \bar{\eta}-m(\tau-\bar{\tau}))} \\
& =e^{\bar{\eta}^{2} / 4 m l^{2}}
\end{aligned}
$$

we find with (102) and (96) that (94) satisfies (81) and (82) provided that the boundary conditions (46) and (47) are satisfied.

A quasi-electron wave function with the correct quasiperiodicity under magnetic translations is hence given by (69) with (49), (94) and (70).

The final form, however, may be simplified significantly. Writing out (94) in full, we have

$$
\begin{aligned}
f_{\bar{\eta}, \partial_{\bar{\eta}}}\left[z, \partial_{z}\right]= & \prod_{i=1}^{N} \vartheta_{\frac{1}{2}, \frac{1}{2}}\left(2 l^{2} \partial_{z_{i}}-\bar{\eta} \mid-\bar{\tau}\right) \\
& \cdot \prod_{i=1}^{N} \exp \left(\frac{\left(z_{i}-2 l^{2} \partial_{z_{i}}+\bar{\eta}-2 m l^{2} \partial_{\bar{\eta}}\right)^{2}}{4 m l^{2} N}\right) \\
& \cdot \exp \left(\mathrm{i} K\left(Z-2 l^{2} \partial_{\bar{\eta}}\right)\right) \\
& \cdot \prod_{\nu=1}^{m} \vartheta_{\frac{1}{2}, \frac{1}{2}}\left(Z-2 l^{2} \partial_{\bar{\eta}}-Z_{\nu} \mid \tau\right) \\
& \cdot \prod_{i<j}^{N} \vartheta_{\frac{1}{2}, \frac{1}{2}}\left(z_{i}-z_{j} \mid \tau\right)^{m} .
\end{aligned}
$$

Note first that the argument of the Gaussian and the argument in the line below commute,

$$
\left[z_{i}-2 l^{2} \partial_{z_{i}}+\bar{\eta}-2 m l^{2} \partial_{\bar{\eta}}, Z-2 l^{2} \partial_{\bar{\eta}}\right]=0,
$$

which implies that we can interchange the orders of $G_{\bar{\eta}, \partial_{\bar{\eta}}}\left[z, \partial_{z}\right]$ and $F_{\partial_{\bar{\eta}}}(Z)$ in (104). Second, since $Q_{\bar{\eta}}\left[\partial_{z}\right]$ and $F_{\partial_{\bar{\eta}}}(Z)$ commute, we may interchange them and thereby bring the derivatives in the $z_{i}$ closer to the Jastrow factor. We may hence write

$$
\begin{aligned}
f_{\bar{\eta}, \partial_{\bar{\eta}}}\left[z, \partial_{z}\right]= & \exp \left(\mathrm{i} K\left(Z-2 l^{2} \partial_{\bar{\eta}}\right)\right) \\
& \cdot \prod_{\nu=1}^{m} \vartheta_{\frac{1}{2}, \frac{1}{2}}\left(Z-2 l^{2} \partial_{\bar{\eta}}-Z_{\nu} \mid \tau\right) \\
& \cdot \prod_{i=1}^{N} \vartheta_{\frac{1}{2}, \frac{1}{2}}\left(2 l^{2} \partial_{z_{i}}-\bar{\eta} \mid-\bar{\tau}\right) \\
& \cdot \prod_{i=1}^{N} \exp \left(\frac{\left(z_{i}-2 l^{2} \partial_{z_{i}}+\bar{\eta}-2 m l^{2} \partial_{\bar{\eta}}\right)^{2}}{4 m l^{2} N}\right) \\
& \cdot \prod_{i<j}^{N} \vartheta_{\frac{1}{2}, \frac{1}{2}}\left(z_{i}-z_{j} \mid \tau\right)^{m} .
\end{aligned}
$$

Substitution of (106) into (69) with (49) and (70) allows now for significant simpification. Since $f_{\bar{\eta}, \partial_{\bar{\eta}}}\left[z, \partial_{z}\right]$ acts on $e^{R}$, and

$$
\begin{aligned}
\left(\bar{\eta}-2 m l^{2} \partial_{\bar{\eta}}\right) e^{\bar{\eta}^{2} / 4 m l^{2}} & =0, \\
\left(z_{i}-2 l^{2} \partial_{z_{i}}\right) e^{z_{i}^{2} / 4 l^{2}} J[z] & =e^{z_{i}^{2} / 4 l^{2}}\left(-2 l^{2} \partial_{z_{i}}\right) J[z],
\end{aligned}
$$

we obtain the final form of the quasi-electron wave function,

$$
\begin{aligned}
\psi_{\bar{\eta}}[z]= & e^{-S} e^{\mathrm{i} K\left(Z-2 l^{2} \partial_{\bar{\eta}}\right)} \\
& \cdot \prod_{\nu=1}^{m} \vartheta_{\frac{1}{2}, \frac{1}{2}}\left(Z-2 l^{2} \partial_{\bar{\eta}}-Z_{\nu} \mid \tau\right) \cdot e^{\bar{\eta}^{2} / 4 m l^{2}} \\
& \cdot \prod_{i=1}^{N} \vartheta_{\frac{1}{2}, \frac{1}{2}}\left(2 l^{2} \partial_{z_{i}}-\bar{\eta} \mid-\bar{\tau}\right) \cdot \prod_{i=1}^{N} e^{z_{i}^{2} / 4 l^{2}} \\
& \cdot \prod_{i=1}^{N} \exp \left(\frac{l^{2} \partial_{z_{i}}^{2}}{m N}\right) \cdot \prod_{i<j}^{N} \vartheta_{\frac{1}{2}, \frac{1}{2}}\left(z_{i}-z_{j} \mid \tau\right)^{m} .
\end{aligned}
$$

where $S$ is given by (49). Since we have not found a plausible way to motivate the form (107) directly, however, we present the results in the order we have obtained them. An additional advantage of (107) over (69) with (104) and (70) is that the generalization to several quasielectrons is straightforward.

This concludes our derivation of the Laughlin's quasielectron states on the torus.

A very valid question to ask at this point is how feasible it is to evaluate (107) explicitly for applications in 
numerical studies. To begin with, the odd Jacobi theta functions in (107) are according to (28) defined as

$$
\vartheta_{\frac{1}{2}, \frac{1}{2}}(z \mid \tau)=\sum_{n \in \mathbb{Z}+\frac{1}{2}} e^{\pi \mathrm{i} n^{2} \tau} e^{\pi \mathrm{i} n} e^{2 \pi \mathrm{i} n z},
$$

where we have adjusted $n$ such that we sum only over half-integer values. If we write $\tau=\tau^{\prime}+\mathrm{i} \tau^{\prime \prime}$, with $\tau^{\prime}, \tau^{\prime \prime} \in \mathbb{R}, \tau^{\prime \prime}>0$, we see that the sum (108) converges the quicker the larger $\tau^{\prime \prime}$. Since the quantum Hall wave functions are modular invariant, with modular transformations generated by

$$
\mathrm{T}: \tau \rightarrow \tau+1 \quad \text { and } \quad \mathrm{S}: \tau \rightarrow-\frac{1}{\tau},
$$

we can always choose a unit cell such that $\tau^{\prime \prime} \geq \frac{1}{2} \sqrt{3}$. For the more typical rectangular unit cells we may even choose $\tau^{\prime \prime} \geq 1$. Even if we assume that $\operatorname{Im}(\mathrm{z})=-\tau^{\prime \prime}$ in (108), we see that the series decays as $e^{-\pi\left(n^{2}-2 n\right) \tau^{\prime \prime}}$, which for $\tau^{\prime \prime}=1$ is of order 1 for $n=\frac{1}{2}$ and of order $10^{-27}$ for $n=\frac{11}{2}$, while the sum of all terms for larger $|n|$ is negligible by comparison. It hence appears sufficient to keep the 12 terms from $n=-\frac{11}{2}$ to $+\frac{11}{2}$. The derivatives in the Jacobi theta functions amount hence only to derivative operators in 12 terms of a sum, which in principle can be evaluated using programs performing symbolic manipulations of mathematical expressions.

A remaining obstacle is posed by the combination of derivatives in the theta functions with the Gaussians in $\bar{\eta}^{2}$ and $z_{i}^{2}$ in the second and third line of (107), respectively, or the Gaussian in the derivatives acting on the arguments of the theta functions in the last line. Using the Baker-Hausdorff formula

$$
e^{x} e^{y}=e^{x+y+\frac{1}{2}[x, y]+\frac{1}{12}[x,[x, y]]+\frac{1}{12}[[x, y], y]},
$$

which truncates as stated if both $[x,[x, y]]$ and $[[x, y], y]$ commute with both $x$ and $y$, we may write

$$
\begin{gathered}
\exp \left(a \partial_{z}^{2}\right) e^{2 \pi \mathrm{i} n z}=e^{2 \pi \mathrm{i} n\left(z+2 a \partial_{z}\right)} \exp \left(a \partial_{z}^{2}\right), \\
e^{2 \pi \mathrm{i} n\left(2 l^{2} \partial_{z}\right)} e^{z^{2} / 4 l^{2}}=e^{z^{2} / 4 l^{2}} e^{2 \pi \mathrm{i} n\left(2 l^{2} \partial_{z}+z\right)}, \\
e^{2 \pi \mathrm{i} n\left(-2 l^{2} \partial_{\bar{\eta}}\right)} e^{\bar{\eta}^{2} / 4 m l^{2}}=e^{\bar{\eta}^{2} / 4 m l^{2}} e^{2 \pi \mathrm{i} n\left(-2 l^{2} \partial_{\bar{\eta}}-\bar{\eta} / m\right)} .
\end{gathered}
$$

Commuting the Gaussian factors through the theta functions will hence only generate terms inside the theta functions which are linear in the arguments of the Gaussian. We may use these commutators to rewrite (107) as

$$
\begin{aligned}
\psi_{\bar{\eta}}[z]= & e^{-S} e^{\bar{\eta}^{2} / 4 m l^{2}} \prod_{i=1}^{N} e^{z_{i}^{2} / 4 l^{2}} \cdot e^{\mathrm{i} K\left(Z-2 l^{2} \partial_{\bar{\eta}}-\bar{\eta} / m\right)} \\
& \cdot \prod_{\nu=1}^{m} \vartheta_{\frac{1}{2}, \frac{1}{2}}\left(Z-2 l^{2} \partial_{\bar{\eta}}-\frac{\bar{\eta}}{m}-Z_{\nu} \mid \tau\right) \\
& \cdot \prod_{i=1}^{N} \vartheta_{\frac{1}{2}, \frac{1}{2}}\left(2 l^{2} \partial_{z_{i}}+z_{i}-\bar{\eta} \mid-\bar{\tau}\right)
\end{aligned}
$$

$$
\cdot \prod_{i<j}^{N} \vartheta_{\frac{1}{2}, \frac{1}{2}}\left(z_{i}-z_{j}+\frac{2 l^{2}\left(\partial_{z_{i}}-\partial_{z_{j}}\right)}{m N} \mid \tau\right)^{m} .
$$

The evaluation of this expression still constitutes a challenge, as each theta function represents a sum of about 12 terms with the argument in exponential functions. Since the arguments now consist of linear functions in the coordinates and derivatives taken in these coordinates, however, the combinations may now be evaluated by repeated application of the Baker-Hausdorff formula, where each commutator gives a contribution which does not depend on the coordinates (but only of the indices $n$ of the sums (108)). Even though we believe that the form (110) is useful for the explicit evaluation of the quasi-electron wave function, we prefer to consider the form (107) as the final result of our derivation. This form displays the desired transformation properties more succinctly.

\section{CONCLUSION}

In this article, we set up an operator formalism for Landau levels and magnetic translations, and used it to formulate Laughlin's wave functions for fractionally quantized Hall states subject to PBCs. The results have been known for three decades for the ground states and the quasi-hole excitations. They are, however, original for the technically more challenging quasi-electron excitations, which were left as an open problem in the classic work by Haldane and Rezayi ${ }^{34}$. Comparing the final form (107)

$$
\begin{aligned}
\psi_{\bar{\eta}}[z]= & e^{-S} e^{\mathrm{i} K\left(Z-2 l^{2} \partial_{\bar{\eta}}\right)} \\
& \cdot \prod_{\nu=1}^{m} \vartheta_{\frac{1}{2}, \frac{1}{2}}\left(Z-2 l^{2} \partial_{\bar{\eta}}-Z_{\nu} \mid \tau\right) \cdot e^{\bar{\eta}^{2} / 4 m l^{2}} \\
& \cdot \prod_{i=1}^{N} \vartheta_{\frac{1}{2}, \frac{1}{2}}\left(2 l^{2} \partial_{z_{i}}-\bar{\eta} \mid-\bar{\tau}\right) \cdot \prod_{i=1}^{N} e^{z_{i}^{2} / 4 l^{2}} \\
& \cdot \prod_{i=1}^{N} \exp \left(\frac{l^{2} \partial_{z_{i}}^{2}}{m N}\right) \cdot \prod_{i<j}^{N} \vartheta_{\frac{1}{2}, \frac{1}{2}}\left(z_{i}-z_{j} \mid \tau\right)^{m}
\end{aligned}
$$

of the quasi-electron wave function with the final form of the quasi-hole wave function

$$
\begin{aligned}
\psi_{\eta}[z]= & e^{-S} e^{\mathrm{i} K(Z+\eta / m)} \\
& \cdot \prod_{\nu=1}^{m} \vartheta_{\frac{1}{2}, \frac{1}{2}}\left(Z+\frac{\eta}{m}-Z_{\nu} \mid \tau\right) \cdot e^{\eta^{2} / 4 m l^{2}} \\
& \cdot \prod_{i=1}^{N} \vartheta_{\frac{1}{2}, \frac{1}{2}}\left(z_{i}-\eta \mid \tau\right) \cdot \prod_{i=1}^{N} e^{z_{i}^{2} / 4 l^{2}} \\
& \cdot \prod_{i<j}^{N} \vartheta_{\frac{1}{2}, \frac{1}{2}}\left(z_{i}-z_{j} \mid \tau\right)^{m}
\end{aligned}
$$


we see that the quasi-electron function is very close to what we would have expected from the known wave functions in the plane, which already contains the derivative operators in the coordinates $z_{i}$ and $\bar{\eta}$ rather than the quasi-electron coordinate $\eta$ in the complex plane. That we need to subtract $2 l^{2} \partial_{\bar{\eta}}$ rather than $\bar{\eta} / m$ or even $\eta / m$ from the center-of-mass coordinate $Z$ is dictated by the quasi-periodic boundary conditions and the requirement that $\psi_{\bar{\eta}}[z]$ is given by $e^{-S}$ times an analytic function in both $z$ and $\bar{\eta}$. The only part we would not have been able to anticipate is the Gaussian factor in the last line of (107). This is a minor modification, which nonetheless required a major effort to obtain.

The final form (107), however, reveals something else we could not have easily anticipated. Since we cannot commute the factors $e^{z_{i}^{2} / 4 l^{2}}$ through the quasi-electron theta function to the right of them without modifying their arguments as indicated in (110), we see that a formulation of the quasi-electron in the otherwise more compact Landau gauge used by Haldane and Rezayi ${ }^{34}$ would require factors $e^{-z_{i}^{2} / 4 l^{2}}$ and $e^{z_{i}^{2} / 4 l^{2}}$ to the left and to the right of this theta function, respectively. This indicates that obtaining the quasi-electron in this gauge would presumably be significantly more difficult, which may explain why the problem has not been solved previously.

We have further seen that the quasi-electron wave function we have derived for PBCs in this article does not lend itself well to explicit evaluation, which sincerely limits the applicability and usefulness of our result. To find out about these limitations, however, it was necessary to obtain the wave function in the first place. The main result of our study may hence be that it is possible to generalize Laughlin's quasi-electron excitation to PBCs, but that it is not practical to work with the ensuing form.

\section{ACKNOWLEDGMENTS}

This work was supported by the ERC starters grant TOPOLECTRICS under ERC-StG-Thomale-336012.

Appendix: Transformation properties of $G_{\bar{\eta}, \partial_{\bar{\eta}}}\left[z, \partial_{z}\right]$

In this appendix, we will sketch the derivation of (92) and (101). To begin with, we introduce the shorthand notation

$$
G_{\bar{\eta}, \partial_{\bar{\eta}}}\left[z, \partial_{z}\right]=G=\prod_{i=1}^{N} G_{i}, \quad G_{i}=\exp \left(\frac{D_{i}^{2}}{4 m l^{2} N}\right)
$$

with $D_{i}=A_{i}+B_{i}, A_{i}=\bar{\eta}-2 l^{2} \partial_{z_{i}}, B_{i}=z_{i}-2 m l^{2} \partial_{\bar{\eta}}$. We then use

$$
\tau-\bar{\tau}=2 \mathrm{i} 2 \pi l^{2} N_{\phi}=2 \pi \mathrm{i} 2 l^{2}(N m-1)
$$

to evaluate

$$
\begin{aligned}
\tilde{t}_{i}(n \tau) G_{i} \tilde{t}_{i}(n \tau)^{-1} \\
=\exp \left(\frac{1}{4 m l^{2} N}\left(\left(D_{i}-2 \pi \mathrm{i} n 2 l^{2}\right)+2 \pi \mathrm{i} n 2 l^{2} m N\right)^{2}\right) \\
=e^{2 \pi \mathrm{i} n A_{i}} e^{2 \pi \mathrm{i} n B_{i}} \\
\quad \cdot \exp \left(\frac{1}{4 m l^{2} N}\left(D_{i}-2 \pi \mathrm{i} n 2 l^{2}\right)^{2}\right) e^{(2 \pi \mathrm{i} n l)^{2}(N m-m-1)} \\
=e^{2 \pi \mathrm{i} n A_{i}} e^{2 \pi \mathrm{i} n\left(-2 l^{2} m \partial_{\bar{\eta}}\right)} G_{i} e^{2 \pi \mathrm{i} n z_{i}} e^{(2 \pi \mathrm{i} n l)^{2}(N m-m-1)} .
\end{aligned}
$$

We proceed

$$
\begin{aligned}
\tilde{t}_{i}(n \tau) G \tilde{t}_{i}(n \tau)^{-1}=\tilde{t}_{i}(n \tau) G_{i} \tilde{t}_{i}(n \tau)^{-1} \prod_{\substack{j=1 \\
(j \neq i)}}^{N} G_{j} \\
=e^{2 \pi \mathrm{i} n A_{i}} e^{2 \pi \mathrm{i} n\left(-2 l^{2} m \partial_{\bar{\eta}}\right)} G e^{2 \pi \mathrm{i} n z_{i}} e^{(2 \pi \mathrm{i} n l)^{2}(N m-m-1)} \\
=e^{2 \pi \mathrm{i} n A_{i}} \prod_{j=1}^{N} \exp \left(\frac{1}{4 m l^{2} N}\left(D_{j}-2 \pi \mathrm{i} n 2 l^{2} m\right)^{2}\right) \\
\cdot e^{2 \pi \mathrm{i} n B_{i}} e^{(2 \pi \mathrm{i} n l)^{2}(N m-m-1)} \\
=e^{2 \pi \mathrm{i} n A_{i}} G e^{-2 \pi \mathrm{i} n D / N} e^{2 \pi \mathrm{i} n B_{i}} e^{\pi \mathrm{i} n^{2}(\tau-\bar{\tau})},
\end{aligned}
$$

where $D$ is given by (93). For $n=1$, this reduces to (92). To verify (101), we write

$$
\begin{gathered}
\tilde{t}_{\bar{\eta}}(m \tau) G \tilde{t}_{\bar{\eta}}(m \tau)^{-1}=\prod_{i=1}^{N} \tilde{t}_{i}(-m \tau) G \prod_{i=1}^{N} \tilde{t}_{i}(-m \tau)^{-1} \\
=\prod_{i=1}^{N} e^{-2 \pi \mathrm{i} m A_{i}} G e^{2 \pi \mathrm{i} m D} \prod_{i=1}^{N} e^{-2 \pi \mathrm{i} m B_{i}} e^{\pi \mathrm{i} m^{2} N(\tau-\bar{\tau})},
\end{gathered}
$$

where we have used

$$
e^{-2 \pi \mathrm{i} m B_{i}} \tilde{t}_{i}(-m \tau) e^{\alpha D} \tilde{t}_{i}(-m \tau)^{-1}=e^{\alpha D} e^{-2 \pi \mathrm{i} m B_{i}}
$$

in obtaining the last line. 
1 R. B. Laughlin, Phys. Rev. Lett. 50, 1395 (1983).

2 F. D. M. Haldane, Phys. Rev. Lett. 51, 605 (1983).

3 B. I. Halperin, Phys. Rev. Lett. 52, 1583 (1984), ibid. 52, E2390 (1984).

4 M. Greiter, Phys. Lett. B 336, 48 (1994).

5 J. Jain, Composite Fermions (Cambridge University Press, Cambridge, 2007).

${ }^{6}$ G. Moore and N. Read, Nucl. Phys. B 360, 362 (1991).

7 M. Greiter, X. G. Wen, and F. Wilczek, Phys. Rev. Lett. 66, 3205 (1991).

8 M. Greiter, X. G. Wen, and F. Wilczek, Nucl. Phys. B 374, 567 (1992).

9 F. Wilczek, Fractional statistics and anyon superconductivity (World Scientific, Singapore, 1990).

10 A. Stern, Annals of Physics 323, 204 (2008).

11 N. Read and D. Green, Phys. Rev. B 61, 10267 (2000).

12 D. A. Ivanov, Phys. Rev. Lett. 86, 268 (2001).

13 A. Stern, Nature 464, 187 (2010).

14 A. Y. Kitaev, Ann. Phys. 303, 2 (2003).

15 C. Nayak, S. H. Simon, A. Stern, M. Freedman, and S. Das Sarma, Rev. Mod. Phys. 80, 1083 (2008).

16 E. Majorana, Nuovo Cimento 14, 171 (37).

17 N. Read and E. Rezayi, Phys. Rev. B 59, 8084 (1999).

18 F. D. M. Haldane, Phys. Rev. Lett. 60, 635 (1988).

19 B. S. Shastry, Phys. Rev. Lett. 60, 639 (1988).

20 M. Greiter, Mapping of Parent Hamiltonians, Vol. 244 of Springer Tracts in Modern Physics (Springer, Berlin/Heidelberg, 2011).

21 R. Thomale, S. Rachel, P. Schmitteckert, and M. Greiter, Phys. Rev. B 85, 195149 (2012).

${ }^{22}$ F. D. M. Haldane, Phys. Rev. Lett. 67, 937 (1991).

${ }^{23}$ M. Greiter, Phys. Rev. B 79, 064409 (2009).

${ }^{24}$ X. G. Wen, Int. J. Mod. Phys. B4, 239 (1990).

25 X. Wen, Quantum Field Theory of Many-Body Systems, Oxford Graduate Texts (Oxford University, New York, 2004).

26 B. A. Bernevig, Topological Insulators and Topological Superconductors (Princeton University Press, Princeton, 2013).

27 V. Kalmeyer and R. B. Laughlin, Phys. Rev. Lett. 59, 2095 (1987).

28 V. Kalmeyer and R. B. Laughlin, Phys. Rev. B 39, 11879 (1989).

29 D. F. Schroeter, E. Kapit, R. Thomale, and M. Greiter, Phys. Rev. Lett. 99, 097202 (2007).

30 R. Thomale, E. Kapit, D. F. Schroeter, and M. Greiter, Phys. Rev. B 80, 104406 (2009).

31 R. Laughlin, Surf. Sci. 142, 163 (1984).

32 G. Fano, F. Ortolani, and E. Colombo, Phys. Rev. B 34, 2670 (1986).

33 M. Greiter, Phys. Rev. B 83, 115129 (2011).

${ }^{34}$ F. D. M. Haldane and E. H. Rezayi, Phys. Rev. B 31, 2529 (1985).

35 M. Greiter and F. Wilczek, Nucl. Phys. B 370, 577 (1992).

36 T. H. Hansson, C.-C. Chang, J. K. Jain, and S. Viefers, Phys. Rev. B 76, 075347 (2007).

37 E. J. Bergholtz, T. H. Hansson, M. Hermanns, and A. Karlhede, Phys. Rev. Lett. 99, 256803 (2007).

38 M. Hermanns, J. Suorsa, E. J. Bergholtz, T. H. Hansson, and A. Karlhede, Phys. Rev. B 77, 125321 (2008).

39 M. Hermanns, Phys. Rev. B 87, 235128 (2013).
40 M. Fremling, T. H. Hansson, and J. Suorsa, Phys. Rev. B 89, 125303 (2014).

41 T. H. Hansson, M. Hermanns, and S. Viefers, Phys. Rev. B 80, 165330 (2009).

42 G. S. Jeon and J. K. Jain, Phys. Rev. B 68, 165346 (2003).

43 B. A. Bernevig and F. D. M. Haldane, Phys. Rev. Lett. 102, 066802 (2009).

44 N. Read, Phys. Rev. B 79, 045308 (2009).

${ }^{45}$ L. Landau, Z. Phys. 64, 629 (1930).

46 D. P. Arovas, Ph.D. thesis, University of California, Santa Barbara, 1986.

47 A. H. MacDonald, Phys. Rev. B 30, 3550 (1984).

48 S. M. Girvin and T. Jach, Phys. Rev. B 28, 4506 (1983).

49 We have not been able to find out who introduced the ladder operators for Landau levels in the plane. The energy eigenfunctions were known since Landau ${ }^{45}$. MacDonald ${ }^{47}$ used the ladder operators in 1984, but neither gave nor took credit. Girvin and $\mathrm{Jach}^{48}$ were aware of two independent ladders a year earlier, but neither spelled out the formalism, nor pointed to references. It appears that the community had been aware of them, but not aware of who introduced them. Clear and complete presentation can be found in Arovas $^{46}$ or Greiter ${ }^{20}$.

50 D. Mumford, Tata Lectures on Theta (Birkhäuser, Basel, 1983), Vol. I and II.

51 G. A. Jones and D. Singerman, Complex Functions (Cambridge University Press, Cambridge, 1987).

52 As a side remark, numerical work on the sphere shows that the quasi-electron wave function can be improved energetically by acting only on a Jastrow factor squared with the derivatives in (67). This is likely to hold for the torus as well. 